\title{
Targets for treatment success
}

\author{
Richard Simon
}

Since the National Cancer Act declaring a war on cancer was signed in December 1971, many effective treatments have been developed for cancer patients. With a few exceptions, however, these successes were not based on a deep understanding of tumor biology. Many of the drugs were developed based on their activity against transplantable mouse tumors or human tumor cell lines. Although the last few decades have been widely heralded for the progress made in understanding tumor biology, these advances have generally not been sufficient for development of effective therapeutics. There are signs that this situation is improving. For example, imatinib was developed to inhibit the constitutively activated BCR-ABL fusion tyrosine kinase for treating chronic myelogenous leukemia, and trastuzumab for blocking the HER2 receptor overexpressed in breast cancer cells.

The past 34 years have seen the development of a vigorous biotechnology industry, and active involvement of the pharmaceutical industry in cancer therapeutics. Jointly they are capable of developing inhibitors of most defined targets. During this time, we have also seen the maturation of an effective infrastructure for conducting high-quality clinical trials for treatment evaluation.

The rate-limiting steps in seeking curative cancer treatment are identification of key molecular targets and development of methods to individualize the right treatment for the right patient. One thing we have learned about tumor biology is that it is easy for tumors to become resistant to almost any drug. Most tumors display extreme genetic heterogeneity, arising from either genetic instability or a large number of cell replications at normal mutation rates. Because of multiple selection pressures that occur during invasion and metastasis, many genetically distinct sublines are generated with characteristic signatures of overexpressed and silenced genes. Since most differentially
For the

development

of curative

treatments that

are nontoxic

to normal

cells, specific

inhibitors

of mutated

oncogenic

protein

products are

an attractive

option.

$R$ Simon is Chief

of the Biometric

Research Branch at

the National Cancer

Institute, Rockville,

$M D, U S A$.

\section{Competing interests}

The author declared he has no competing interests.

www.nature.com/clinicalpractice doi:10.1038/ncponc0402 expressed proteins are characteristic of only a subset of the tumor cells, regulation of such targets often merely selects for overgrowth by other tumor cells. Depending on the predominance of the target, the treatment might be partially effective, but not curative.

Resistance might be avoided by targeting the key early oncogenic mutations that should be present in all tumor cells. A treatment that specifically inhibits the mutant protein product could be effective against all tumor cells and nontoxic to normal cells. One might still select for cells that are resistant to a given drug because a subsequent mutation interferes with target binding, but that type of resistance is more easily overcome, as demonstrated with imatinib. This strategy, if combined with innovative drug-development strategies that emphasize early treatment, could greatly enhance chances of success. Although oncogenesis usually requires more than one mutational event, this does not mean that multiple dysregulated pathways need to be corrected to reverse the malignant phenotype, as experimental evidence has shown.

For the development of curative treatments that are nontoxic to normal cells, specific inhibitors of mutated oncogenic protein products are an attractive option. Implementing such a strategy, however, requires key oncogenicmutation identification. This is different to identifying all mutations in the dominant clones of relatively advanced tumors and innovative approaches should be explored. Discovery of a point mutation in the BRAF gene, which is present in over $60 \%$ of human melanoma tumors and appears to drive early tumor growth, is encouraging for this approach. The time is ripe to consider this and other strategies for identification of key molecular targets. Many of the current therapeutic targets do not appear sufficiently specific for cancer cells, and it is important to establish more-stringent standards for therapeutic targets. 